\title{
The Influence of Intra-Team Communication on the Attacking Success in Rugby
}

\author{
Ika Novitaria Marani*, Iwan Barata, Rendi Gunawan \\ Fakultas Ilmu Olahraga \\ Universitas Negeri Jakarta \\ Jakarta, Indonesia \\ *ika.novitaria@unj.ac.id
}

\begin{abstract}
In game sports, such as rugby, communication is very important because, in game sports, there are signs or shouts so that their team gets a victory. Communication in a competition is needed to avoid mistakes that might occur because communication has information that must be conveyed from one player to another player in a coherent, true, and clear manner. With communication, a team delivers a message that is expected can be done by a teammate, so that a good cooperation occurs within the team. Therefore, this study had the aim to find out the effect of intra-team communication on the success of attacking in rugby sport. The method used was an associative method. The study was conducted at the 2018 Jak10 Series match. The population of this study were the male rugby athletes of Jakarta State University who participated in the 22 rugby National Championship. The sampling technique used was total sampling technique, so that the sample of this study were all 22 male rugby athletes of Jakarta State University who participated in the Rugby National Championship. The results of this study showed that the correlation coefficient $r y=0.628$, the value of count $=$ 3.609 , and $t$-Table $=2.08$ with the significance level at 0.05 . This indicates that intra-team communication influences the success of attacking in rugby sport.
\end{abstract}

Keywords: intra-team communication, rugby, attacking success

\section{INTRODUCTION}

The goal of a team sport is how to score as many points as possible. Likewise, the aim of the rugby team is to score points by crossing the opponent's goal line (bottom line) and making the ball touch the ground when in contact with it. To be able to get points, the team must create space and attack when the opponent's defence weakens. This indicates that a team is very important to have effective principles that can be used to score points in a match. For example, having good and fast attack tactics by creating space for the attacker to create numbers. This is as stated by Wheeler that: The ability of the attacking ball carrier to receive the ball at high speed with at least two body lengths from the defence line against an isolated defender promoted tackle-breaks [1].

To be able to create space and opportunities that are good and fast, it is necessary to build a team that has good cohesiveness. But to build a team that has good cohesiveness is an ongoing and diverse process, because group members must learn how to work together to achieve common goals by sharing information related to the function of each group member in order to be able to build a way to achieve the goal of obtaining numbers as much as possible - and get a victory. To be able to achieve the goals of the game, cooperation with others is very important. The role of a sports team coach is to encourage team members to work together, teach social skills and strengthen team spirit. During matches in sports competitions, specific languages are visual and audible movement codes. Its success depends on the relationship of tactical teammates who are consolidated and synchronize their actions. Under time pressure and rapid game development, players must make decisions that are synchronized tactically. In this context nonverbal communication is common because during games, except for time outs, there is no time for words. Relationships between teammates are also socially meaningful and sometimes emotionally. Nonverbal communication usually carries more emotional meaning than words alone [2]. This was also expressed by Yukelson that: Core components to consider in building a successful team include having a shared vision and unity of purpose, collaborative and synergistic teamwork, individual and mutual accountability, an identity as a team, a positive team culture and cohesive group atmosphere [3]. Open and honest communication processes, peer helping and social support, and trust at all levels.

Therefore, in order to win, it is necessary to create a group that has good cohesiveness. To be able to have a group that has good cohesiveness one of the keys is to have good communication between teams. This is supported by McLaren which says that interaction and communication as one of the processes that affect cohesion [4]. Because by increasing interaction and communication team members can help understand how individuals see sports teams more cohesively. In addition, Smith also stated that communication is a potential mechanism that might explain the processes through which leadership develops task cohesion [5]. These studies show that communication contributes to team cohesiveness. Where, communication as a key process that facilitates bonding between group members.

Why is communication between team members important? Because effective communication will be seen when team members listen to each other and try to build their respective contributions. In team situations, where disputes might occur, the ability to recognize and resolve conflicts is important for team success. The creation of feelings of trust, respect and understanding is a key element in team development $[6,7]$. 
However, research conducted on the effect of intra-team communication on team sports is very lacking. Even though team sports in Indonesia are starting to develop like rugby. However, team sports achievements in Indonesia have not given maximum results. So that, researchers felt interested in conducting research on the effect of intra-team communication on the success of attacking rugby sports. In order to find out how the influence of intra-team communication on the success of an attack is strengthened by data analysis.

\section{ReSEARCh Methodology}

\section{A. Data Analysis Method and Technique}

This research method used in this study is the associative method. This research was conducted by taking data by direct survey to the field. By observing the rugby team that is competing.

This study uses data analysis techniques using descriptive and inferential statistics. Descriptive statistics are associated with applying statistical methods to collect, process, present and analyse quantitative data descriptively. Whereas inferential statistics are data analysis techniques using statistics by making general conclusions.

\section{B. Subject}

Data is collected when there is a Jak10 Series Championship in Jakarta. The population in this study is the male rugby athlete of Jakarta State University who participated in the National Rugby Championship amounting to 22 people. The sampling technique used is total sampling. So that the sample or research subjects used were male rugby athlete Jakarta State University as many as 22 people.

\section{Instrument Test}

The test instruments used in the study used two types of instruments, namely: test instruments for data collection about the effectiveness of intra-team communication and test instruments for data collection about the success of attacking in rugby sports. The instrument test that uses the measure intrateam communication using a questionnaire with a Likert scale score that has 5 answer choices, namely: Strongly agree, agree, doubt, disagree and strongly disagree. And the instrument test that uses to measure the success of attacks in rugby using empty table that will be filled with the observations of the ability the rugby's team to produce points.

\section{RESULTS AND DISCUSSION}

\section{A. Results}

The results of the study consisted of two data, namely descriptive data and inferential data. Descriptive data contains a description of data that aims to provide a general description of the distribution of data in the form of a frequency distribution location size that is average, maximum value, minimum value, standard deviation, and mode, median. While inferential data contains calculations that are used to test hypotheses so as to make conclusions to be generalized. The results of research based on descriptive data can be seen in the table below:
TABLE I. DESCRIPTION OF RESEARCH RESULTS

\begin{tabular}{|l|l|l|}
\hline \multirow{2}{*}{ Description } & \multicolumn{2}{|c|}{ Variable } \\
\cline { 2 - 3 } & Communication Intra-team & Attack Success \\
\hline Median & 112.50 & 24 \\
\hline Modus & 112 & 24 \\
\hline Mean & 113.1 & 22 \\
\hline $\begin{array}{l}\text { Standar } \\
\text { deviastion }\end{array}$ & 4.62 & 8.07 \\
\hline
\end{tabular}

The following is a table of the frequency of the two research variables, namely: communication between personal and the success of attack on rugby

TABLE II. COMMUNICATION INTRATEAM DISTRIBUTION FREQUENCY

\begin{tabular}{|c|c|c|c|}
\hline \multirow{2}{*}{ No } & \multirow{2}{*}{ Interval Class } & \multicolumn{2}{|c|}{ Frequency } \\
\hline & & Absolut & Relative (\%) \\
\hline 1 & $34.68-38.22$ & 5 & $23 \%$ \\
\hline 2 & $38.23-45.02$ & 2 & $9 \%$ \\
\hline 3 & $45.03-51.82$ & 4 & $18 \%$ \\
\hline 4 & $51.83-58.62$ & 5 & $23 \%$ \\
\hline 5 & $58.63-65.42$ & 6 & $27 \%$ \\
\hline & Total & 22 & $100 \%$ \\
\hline
\end{tabular}

TABLE III. FREQUENCY Distribution OF SUCCESS ATtACKING RUGBY

\begin{tabular}{|c|c|c|c|}
\hline \multirow{2}{*}{ No } & \multirow{2}{*}{ Interval Class } & \multicolumn{2}{|c|}{ Frequency } \\
\hline & & Absolut & $\begin{array}{c}\text { Relative } \\
(\%)\end{array}$ \\
\hline 1 & $31.43-37.16$ & 2 & $9 \%$ \\
\hline 2 & $37.17-42.90$ & 3 & $14 \%$ \\
\hline 3 & $42.91-48.64$ & 2 & $9 \%$ \\
\hline 4 & $48.65-54.38$ & 9 & $41 \%$ \\
\hline 5 & $54.39-62.47$ & 6 & $27 \%$ \\
\hline & Total & 22 & $100 \%$ \\
\hline
\end{tabular}

Based on the results of testing the research hypothesis, the regression equation can be $\bar{Y}=0.02+0.24 \mathrm{X}$. The correlation coefficient must be tested in advance about its significance, before it is used to draw conclusions. The results of the correlation coefficient test can be seen in the following table:

TABle IV. Significance Test Correlation CoEfFicient (X) To (Y)

\begin{tabular}{|l|l|l|}
\hline \multicolumn{1}{|c|}{ Coefficient correlation } & \multicolumn{1}{|c|}{ t. count } & \multicolumn{1}{|c|}{ t. Table } \\
\hline 0,628 & 3,609 & 2,08 \\
\hline
\end{tabular}

From the test of significant of the correlation coefficient above shows that tcount $=3.609$ is greater than table $=2.08$ means the correlation coefficient ry $=0.628$ is significant. This means that the research hypothesis which states that there is an influence between intra-team communications with attack success is accepted. The determinant coefficient for intra-team communication with attack success was 0.394 . This also means that $39.39 \%$ of successful attacks are determined by intra-team communication. 


\section{B. Discussion}

Based on the hypothesis that has been statistically tested, there is an influence between communication intra-team with the success of attacking rugby athletes at Jakarta State University. This shows that if the intra-team communication of a rugby athlete is good then the results of the success of attacking a rugby athlete will also be good, and vice versa if the inter-team communication of an athlete is bad so the results of successful attack will also be bad. This shows that to get success in carrying out attacks requires effective communication between team members. This is also supported by research conducted by [8] which states that: Effective communication has been defined as those interactions between teammates that result in enhanced team attributes and/or functioning. According to the conceptual framework of Social Exchange Theories, these exchanges should result in a wide variety of outcomes.

Effective communication is seen when teammates listen to each other and try to build their respective contributions. Therefore, providing effective communication knowledge will bring a good relationship between the coach and the athlete so that it can contribute to the athlete's performance. Making effective communication paves the way for learning new skills with a higher level of quality through receiving clear feedback about the execution of the athlete's skills. The process of making communication and transferring knowledge to athletes should not be based on instinctive communication patterns but in a more efficient way [9].

Communication between teammates is very important when playing rugby. Communication can be done through the cues and symbols given by fellow team members to deliver the intended message and of course can be understood by the person receiving the message. With communication, a team delivers a message that we hope can be done by a teammate, so that good cooperation occurs within the team. If in a team there is no good communication, there will often be communication errors, Examples of communication errors that can occur are the printing of numbers due to lack of cooperation between the defender and attacking players. So we need an intensive and ongoing communication. This is supported by research conducted by [10] which states that: it is important to maintain high frequency in communication in the field to be able to motivate team members, organize and coordinate their movements during play.

Based on the description, communication with the coordination of gestures is the ability to combine the senses of the speaker with the level of success in the process of producing points. Therefore, communication depends on our ability to be able to understand one another (communication depends on our ability to understand one another). Because the main source of misunderstanding in communication is the way the recipient captures the meaning of a message differently from what was sent by the sender, because the sender failed to communicate his intentions correctly. Resulting in an ineffectiveness in communication. Ineffectiveness in communication can lead individuals to dislike, lose confidence, refuse to listen and disagree with each other's opinions, which of course will result in a large number of interpersonal relations problems.

\section{REFERENCES}

[1] K.W. Wheeler, C.D. Askew, M.G. Sayers, "Effective attacking strategies in rugby union," Eur J Sport Sci, vol. 10, pp. 237-242, 2010.

[2] C.L. Pop, and M.V. Zamfir, "Nonverbal communication of young players in team sports, Pedagogy of Physical Culture and Sports,vol. 24(1), pp.26-29, 2020.

[3] D. Yukelson, "Journal of Applied Sport Psychology Principles of effective team building interventions in sport: A direct services approach at Penn State University Principles of Effective Team Building Interventions in Sport: A Direct Services Approach at Penn State," Journal of Applied Sport Psychology, vol.9(1), pp.73-96, 1997.

[4] C.D. McLaren, and K.S. Spink, "Team Member Communication and Perceived Cohesion in Youth Soccer," Commun Sport,vol. 6, pp. 111125, 2018.

[5] M.J. Smith, C.A. Arthur, J. Hardy, et al, "Transformational leadership and task cohesion in sport: The mediating role of intrateam communication," Psychol Sport Exerc, vol. 14, pp. 249-257, 2013.

[6] P.A. Sullivan, "Communication Skills Training for Interactive Sports," Sport Psychol, vol. 7, pp. 79-91, 2016.

[7] P.J. Sullivan, and N. Callow, "A Cross-Cultural Examination of the Factor Structure of the Scale for Effective Communication in Team Sports," Group Dynamics: Theory, Research, and Practice, vol. 9(2), p. 87, 2005.

[8] P.J. Sullivan, and C.J. Gee, "The Relationship Between Athletic Satisfaction and Intrateam Communication," Gr Dyn, vol. 11, pp. 107116, 2007.

[9] M. Savardelavar, G. Kuan and N. Chin, "Making effective communication with athletes," Coaching Journal, vol. 2, p. 4, 2017.

[10] A. LeCouteur, and R. Feo, "Real-time communication during play: Analysis of team-mates' talk and interaction," Psychol Sport Exerc, vol. 12, pp. 124-134, 2011. 